\title{
Airway pressure release ventilation in mechanically ventilated patients with COVID-19: a multicenter observational study
}

\author{
John S. Zorbas', Kwok M. Ho ${ }^{2,3,4}$, Edward Litton ${ }^{3,5}$, Bradley Wibrow ${ }^{1,3}$, Edward Fysh ${ }^{6}$, Matthew H. Anstey ${ }^{1,3}$ \\ ${ }^{1}$ Intensive Care Department, Sir Charles Gairdner Hospital, Perth; ${ }^{2}$ Intensive Care Department, Royal Perth Hospital, Perth; ${ }^{3}$ University of Western Australia \\ School of Medicine, Perth; ${ }^{4}$ School of Veterinary \& Life Sciences, Murdoch University, Perth; ${ }^{5}$ Intensive Care Department, Fiona Stanley Hospital, Perth; \\ ${ }^{6}$ Intensive Care Department, St John of God Midland Hospital, Perth, Australia
}

Background: Evidence prior to the coronavirus disease 2019 (COVID-19) pandemic suggested that, compared with conventional ventilation strategies, airway pressure release ventilation (APRV) can improve oxygenation and reduce mortality in patients with acute respiratory distress syndrome. We aimed to assess the association between APRV use and clinical outcomes among adult patients receiving mechanical ventilation for COVID-19 and hypothesized that APRV use would be associated with improved survival compared with conventional ventilation. Methods: A total of 25 patients with COVID-19 pneumonitis was admitted to intensive care units (ICUs) for invasive ventilation in Perth, Western Australia, between February and May 2020. Eleven of these patients received APRV. The primary outcome was survival to day 90 . Secondary outcomes were ventilation-free survival days to day 90 , mechanical complications from ventilation, and number of days ventilated.

Results: Patients who received APRV had a lower probability of survival than did those on other forms of ventilation (hazard ratio, $0.17 ; 95 \%$ confidence interval, $0.03-0.89 ; \mathrm{P}=0.036$ ). This finding was independent of indices of severity of illness to predict the use of APRV. Patients who received APRV also had fewer ventilator-free survival days up to 90 days after initiation of ventilation compared to patients who did not receive APRV, and survivors who received APRV had fewer ventilator-free days than survivors who received other forms of ventilation. There were no differences in mechanical complications according to mode of ventilation.

Conclusions: Based on the findings of this study, we urge caution with the use of APRV in COVID-19.

Key Words: COVID-19; intensive care; respiration, artificial; ventilation mode, APRV

\section{INTRODUCTION}

Coronavirus disease 2019 (COVID-19) is a rapidly developing disease caused by the novel severe acute respiratory syndrome coronavirus 2 (SARS-CoV-2) betacoronavirus [1]. On the March 11, 2020, the World Health Organization declared COVID-19 to be a pandemic and a public health crisis [2]. As of August 23, 2020, there have been more than 23 million cases of COVID-19 worldwide and 800,000 reported deaths [3]. Most patients infected with SARSCoV-2 experience a mild illness, with upper respiratory tract infection symptoms that resolve

\section{Original Article}

Received: January 4, 2021 Revised: March 1, 2021

Accepted: March 17, 2021

Corresponding author Matthew H. Anstey Intensive Care Department, Sir Charles Gairdner Hospital, Hospital Ave, Perth 6009, Australia

Tel: +61-864571010

E-mail: Matthew.Anstey@health. wa.gov.au

Copyright $(\bigodot 2021$ The Korean Society of Critical Care Medicine

This is an Open Access article distributed under the terms of Creative Attributions Non-Commercial License (https:// creativecommons.org/li-censes/by-nc/4.0/ which permits unrestricted noncommercial use, distribution, and reproduction in any medium, provided the original work is properly cited. 
with a subsequently uneventful recovery $[4,5]$. However $14 \%$ of patients will experience severe disease (dyspnoea, tachypnoea, hypoxaemia, arterial oxygen partial pressure $\left(\mathrm{PaO}_{2}\right)$ to fractional inspired oxygen $\left(\mathrm{FiO}_{2}\right)$ ratio $<300$, and/or lung infiltrates), and $5 \%$ will develop critical illness (respiratory failure, septic shock, multi-organ dysfunction) [6]. The mortality for patients requiring invasive ventilation due to COVID-19 is extremely high at $83 \%[7,8]$.

Prior research has suggested that, in adult patients who require mechanical ventilation for hypoxic respiratory failure, airway pressure release ventilation (APRV) is associated with a mortality benefit and improved oxygenation compared to conventional ventilation strategies [9]. APRV inverts the respiratory cycle, with the majority occurring in inspiration at a higher set pressure $\left(\mathrm{P}_{\text {high }}\right)$, followed by a brief period of lower pressure $\left(\mathrm{P}_{\text {low }}\right)$. This allows increased lung recruitment (higher mean airway pressures over the respiratory cycle), improved ventilation/perfusion matching, and reduced atelectrauma (shear stress from opening/closing alveoli) [10]. Furthermore, a recent randomised control trial suggested that early use of APRV in mechanically ventilated patients with acute respiratory distress syndrome (ARDS) was associated with an increase in number of ventilator-free days, an increase in successful extubations, a decrease in requirement for tracheostomy, and a decrease in intensive care unit (ICU) mortality [11]. Currently, there is very limited published research on ventilation strategies for COVID-19 pneumonitis.

The primary objective of this study was to assess the association between APRV use and clinical outcomes among adult ICU patients receiving mechanical ventilation for COVID-19 pneumonitis. Our hypothesis was that use of APRV would improve outcomes in patients with COVID-19 pneumonitis compared to other forms of invasive mechanical ventilation.

\section{MATERIALS AND METHODS}

\section{Study Design and Participants}

This was a multicenter, observational study across ICUs in five tertiary and urban district hospitals in Western Australia. Design and the majority of data collection were prospective, although data for the first three patients were collected retrospectively due to delay for ethics approval. Ethics approval and waiver of consent were obtained from the Human Research Ethics Committees of Sir Charles Gairdner Hospital (RGS0000004003), St. John of God Midland (\#1670), and Ramsay Health (\#2020.074). All adult patients who were admitted to the ICU with a clinical diagnosis of COVID-19 pneumonitis

\section{KEY MESSAGES}

- Use of airway pressure release ventilation (APRV) in severe coronavirus disease 2019 (COVID-19) respiratory failure might be associated with longer duration of mechanical ventilation and worse survival outcomes compared with conventional modes of mechanical ventilation.

- Clinicians should be cautious in the use of APRV in mechanically ventilated patients with COVID-19.

and with a positive polymerase chain reaction result for SARSCoV-2 in a sputum or nasopharyngeal swab between February and May 2020 were included in the study. Patients who did not receive invasive mechanical ventilation were excluded from analysis. No further exclusions were applied to minimize selection bias. Data were collected until the final hospital discharge of the cohort on June 19, 2020.

\section{Data Collection}

Patient data were collected from respective medical records with a case report form to minimize information bias. Baseline data included age, sex, weight, date/time of admission, symptoms, smoking history, pregnancy status, current medications, past medical history, Australia and New Zealand Intensive Care Society frailty score, source of admission to ICU, Acute Physiology and Chronic Health Evaluation (APACHE) II score and diagnostic code, physiological support at time of admission to ICU, presence of infiltrates on chest X-ray, date/ time of intubation, and mode and settings of ventilation at admission to ICU. Use of APRV at any time placed that patient in the "APRV" group, and the comparator group comprised patients who received any other mode of mechanical ventilation.

Daily data were collected, including use of pulmonary vasodilators, neuromuscular blockade, prone positioning, disease modifying treatments for COVID-19, and occurrence of ventilator-associated lung injury (pneumothorax, pneumomediastinum, surgical emphysema, and intercostal catheter placement). Twice daily data were collected on mode of ventilation, ventilator settings/parameters (tidal volume, $\mathrm{FiO}_{2}$, $\mathrm{P}_{\text {high }} /$ peak inspiratory pressure, $\mathrm{P}_{\text {low }} /$ positive end-expiratory pressure, lung compliance, time $[\mathrm{T}]_{\text {high }} / \mathrm{T}_{\text {insp }}, \mathrm{T}_{\text {low }}$ ), patient observations (heart rate, respiratory rate, mean arterial pressure, central venous pressure), and use of noradrenaline.

Outcome measures including date and time of ventilation cessation, ICU survival, hospital survival, re-admission to ICU, total length of stay, and tracheostomy use were collected. Study 
data were managed using REDCap electronic data capture tools [12,13].

\section{Outcomes}

Primary outcome was survival to day 90 . Secondary outcomes were number of ventilation-free survival days to day 90 , mechanical complications from ventilation, and number of days ventilated. Mechanical complications from ventilation included pneumothorax, pneumomediastinum, surgical emphysema, and need for intercostal catheter placement. Further outcomes examined were hospital mortality, hospital length of stay, ICU mortality, ICU length of stay, need for tracheostomy, and need for extracorporeal membrane oxygenation (ECMO).

\section{Statistical Analysis}

Continuous data are presented as median (with interquartile range), and categorical data are presented as total (with percentage). We assessed the significance of differences in continuous variables between survivors and non-survivors using the Mann-Whitney test, and we used the chi-square test to assess the significance of differences in categorical variables between survivors and non-survivors. We also assessed differences in concurrent treatments and outcomes between those treated and untreated with APRV using the Mann-Whitney test for continuous variables and the chi-square test for categorical variables. Tests were two-tailed, and a P-value less than 0.05 indicated statistical significance. Stepwise Cox proportional hazards regression was used to assess the effect of exposure to APRV on ICU mortality. Propensity scoring based on severity of illness was used to predict the use of APRV to reduce confounding effects. Statistical analysis was performed using the Stata 12 (StataCorp., College Station, TX, USA).

\section{RESULTS}

Over the study period, 29 patients admitted to the ICU who met the inclusion criteria were included in the study. Of those 29 patients, four were not mechanically ventilated and were excluded from analysis. Initiation of mechanical ventilation was at the discretion of the treating intensive care or emergency specialist in the participating hospital, usually based on a number of factors including oxygen requirement and work to breathe. Of the 25 patients that were invasively ventilated, nine were initially treated with synchronized intermittent mechanical ventilation, 11 with Bilevel, three with APRV, and two with pressure support (Figure 1). There were no significant differences in the baseline characteristics of hospital survivors and non-
29 Patients admitted to ICU from February 2020 to May 2020

Excluded

4 Patients not invasively mechanically ventilated

25 Patients included in the study

Figure 1. Flowchart of patients included in the study. ICU: intensive care unit.

survivors, except for hospital length of stay (Table 1). Ventilation parameters of $\mathrm{FiO}_{2}$, first measured peak inspiratory pressure, first $\mathrm{PaO}_{2}$ available on arterial blood gas, first $\mathrm{PaCO}_{2}$ available on arterial blood gas, and first $\mathrm{PaO}_{2} / \mathrm{FiO}_{2}$ ratio did not differ. Of note, median compliance of all invasively ventilated patients was $43 \mathrm{ml} / \mathrm{cm} \mathrm{H}_{2} \mathrm{O}$ (IQR, 29-50).

Ventilator-free survival days to day 90 were lower in the group treated with APRV than in those who did not receive APRV (Table 2). One of the patients receiving APRV developed pneumothorax, which required an intercostal catheter. There were no differences in concurrent treatments between patients treated with or not treated with APRV (Table 3). No patient received renal replacement therapy, remdesivir, tocilizumab, anakinra, interferon $1 \mathrm{~b}$, or ECMO.

The APRV group had a lower probability of survival to day 90 compared to the non-APRV group of mechanically ventilated patients, with a hazard ratio of survival of 0.17 (95\% confidence interval, 0.03-0.89; $\mathrm{P}=0.036$ ) (Figure 2). Propensity scoring based on indices of severity of illness (APACHE II score, $\mathrm{PaO}_{2} / \mathrm{FiO}_{2}$ ratio, maximum C-reactive protein level, respiratory compliance at initiation of mechanical ventilation, number of quadrants on chest X-ray on admission to ICU, and $\mathrm{FiO}_{2}$ ) were not predictive of APRV (Table 4). Furthermore, a stepwise Cox proportional hazards regression with indices of severity of illness did not change the magnitude or direction of the adverse association between ICU mortality and APRV (Table 5). This reduced probability of ICU survival remained unchanged after adjusting for overfitting bias using bootstrap sampling of 1,000 resamples.

Higher driving pressures over 10 days following initiation of mechanical ventilation were seen in the APRV group than in patients who did not receive APRV (Figure 3). Additionally, lower respiratory compliance was seen in the APRV group than the non-APRV group over a 10-day period with comparisons made every 12 hours (Figure 4 ). 
Table 1. Differences in baseline characteristics between patients treated and untreated with APRV $(n=25)$

\begin{tabular}{|c|c|c|c|}
\hline Baseline characteristics & Treated with APRV $(n=11)$ & Untreated with APRV $(n=14)$ & P-value \\
\hline Age (yr) & 70 (69-77) & $72(69-74)$ & 0.647 \\
\hline Male sex & $8(73)$ & $6(43)$ & 0.227 \\
\hline Weight (kg) & $84(78-119)$ & $83(68-95)$ & 0.373 \\
\hline $\mathrm{BMI}\left(\mathrm{kg} / \mathrm{m}^{2}\right)$ & $31(28-46)$ & $27(23-37)$ & 0.138 \\
\hline Day of symptoms before admission & $6(3-10)$ & $8(3-14)$ & 0.709 \\
\hline Hypertension & $10(46)$ & $6(86)$ & 0.093 \\
\hline Diabetes mellitus & $3(27)$ & $5(36)$ & 0.999 \\
\hline No. of quadrants with infiltrates on CXR & $3(2-4)$ & $4(3-4)$ & 0.467 \\
\hline APACHE II score & $16(12-26)$ & $17(13-20)$ & 0.467 \\
\hline First $\mathrm{PaO}_{2} / \mathrm{FiO}_{2}$ ratio $(\mathrm{mm} \mathrm{Hg})$ & $151(72-178)$ & $157(137-257)$ & 0.347 \\
\hline First peak inspiratory pressure $\left(\mathrm{cm} \mathrm{H}_{2} \mathrm{O}\right)$ & $24(22-29)$ & $22(20-26)$ & 0.267 \\
\hline First PEEP $\left(\mathrm{cm} \mathrm{H}_{2} \mathrm{O}\right)$ & $12(1-12)$ & $10(10-12)$ & 0.851 \\
\hline First respiratory compliance $\left(\mathrm{ml} / \mathrm{cm} \mathrm{H}_{2} \mathrm{O}\right)$ & $32(23-49)$ & $46(35-50)$ & 0.222 \\
\hline
\end{tabular}

Values are presented as median (IOR) or number (\%).

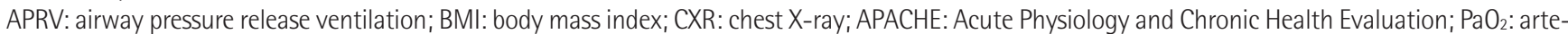
rial oxygen partial pressure; $\mathrm{FiO}_{2}$ : fractional inspired oxygen; PEEP: positive end-expiratory pressure; IQR: interquartile range.

aMedian time to initiation of APRV from initiation of invasive mechanical ventilation was 1 day $(I 0 R, 0-4 ; 4$ patients on day 0,3 patients on day 1,1 patient each on days $3,4,5$, and 6), and the median duration of APRV use was 7 days (IOR, 6-12); ${ }^{\circ}$ Categorical data (male sex, hypertension, diabetes mellitus) were analyzed by chi-square test and continuous data (all others) were analyzed by Mann-Whitney test.

Table 2. Differences in outcomes between patients treated and untreated with APRV $(n=25)$

\begin{tabular}{lccc}
\hline Outcome & $\begin{array}{c}\text { Treated } \\
\text { with APRV } \\
(\mathrm{n}=11)\end{array}$ & $\begin{array}{c}\text { Untreated } \\
\text { with APRV } \\
(\mathrm{n}=14)\end{array}$ & $\begin{array}{c}\text { P- } \\
\text { value }^{b}\end{array}$ \\
\hline Pneumothorax & $1(9)^{c}$ & 0 & 0.440 \\
Subcutaneous emphysema & $1(9)^{c}$ & 0 & 0.440 \\
Intercostal catheter & $1(9)^{c}$ & 0 & 0.440 \\
Tracheostomy & $2(18)$ & $1(7)$ & 0.565 \\
ICU mortality & $5(46)$ & $1(7)$ & 0.056 \\
Hospital mortality & $5(46)$ & $1(7)$ & 0.056 \\
Day 90 mortality & $5(46)$ & $1(7)$ & 0.056 \\
Ventilator-free survival day up to day & $57(1-72)$ & $82(73-85)$ & 0.001 \\
90 after initiation of ventilation & & & \\
Ventilator-free day among survivors & $72(65-78)$ & $82(76-85)$ & 0.017 \\
Total mechanical ventilation day & $12(7-23)$ & $8(4-13)$ & 0.107 \\
ICU stay (day) & $20(8-27)$ & $11(7-15)$ & 0.120 \\
Hospital stay (day) & $33(7-42)$ & $19(14-32)$ & 0.609 \\
Hydroxychloroquine & 0 & $2(14)$ & 0.191 \\
\hline
\end{tabular}

Values are presented as number (\%) or median (IQR).

APRV: airway pressure release ventilation; ICU: intensive care unit; IQR: interquartile range.

aMedian time to initiation of APRV from initiation of invasive mechanical ventilation was 1 day $(I Q R, 0-4 ; 4$ patients on day 0,3 patients on day 1, 1 patient each on days $3,4,5$, and 6$)$, and median duration of APRV use was 7 days (IQR, 6-12); ${ }^{b}$ Categorical data (pneumothorax, subcutaneous emphysema, intercostal catheter, tracheostomy, mortality, hydroxychloroquine) were analyzed by chi-square test and continuous data (all others) were analyzed by Mann-Whitney test; ' ${ }^{\circ}$ cccurred 3 days after APRV was initiated in the same patient.
Table 3. Differences in concurrent treatments for patients treated and untreated with APRV $(n=25)$

\begin{tabular}{lccc}
\hline Concurrent treatment & $\begin{array}{c}\text { Treated with } \\
\text { APRV }^{a} \\
(n=11)\end{array}$ & $\begin{array}{c}\text { Untreated } \\
\text { with APRV } \\
(n=14)\end{array}$ & $\begin{array}{c}\text { P- } \\
\text { value }^{b}\end{array}$ \\
\hline Azithromycin & $10(91)$ & $11(79)$ & 0.604 \\
Lopinavir/ritonavir & 0 & $2(14)$ & 0.174 \\
Corticosteroids & $3(27)$ & $4(29)$ & 0.943 \\
Vitamin C & $1(9)$ & 0 & 0.250 \\
Prone ventilation & $5(46)$ & $1(7)$ & 0.056 \\
Day to initiation of prone ventila- & $3(3-5)$ & $2(\mathrm{NA})$ & 0.333 \\
$\quad$ tion from initiation of ventilation & & & \\
Nitric oxide & $5(46)$ & $1(7)$ & 0.050 \\
Day to initiation of nitric oxide & $3(2-5)$ & $4(\mathrm{NA})$ & 0.667 \\
Prostacyclin & $3(27)$ & 0 & 0.072 \\
Day to initiation of prostacyclin & $3(2-\mathrm{NA})$ & $\mathrm{NA}$ & $\mathrm{NA}$ \\
\hline
\end{tabular}

Values are presented as number (\%) or median (IOR). No patient was treated with renal replacement therapy, remdesivir, tocilizumab, anakinra, interferon $1 b$, or extracorporeal membrane oxygenation.

APRV: airway pressure release ventilation; NA, not applicable; IQR: interquartile range.

aedian time to initiation of APRV from initiation of invasive mechanical ventilation was 1 day $(I Q R, 0-4 ; 4$ patients on day 0,3 patients on day $1,1$ patient each on days $3,4,5$, and 6$)$, and median duration of APRV

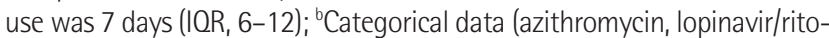
navir, corticosteroids, vitamin C, prone ventilation, nitric oxide, prostacylin) were analyzed by chi-square test and continuous data (all others) were analyzed by Mann-Whitney test; ${ }^{C}$ Data from 24 patients were available. 


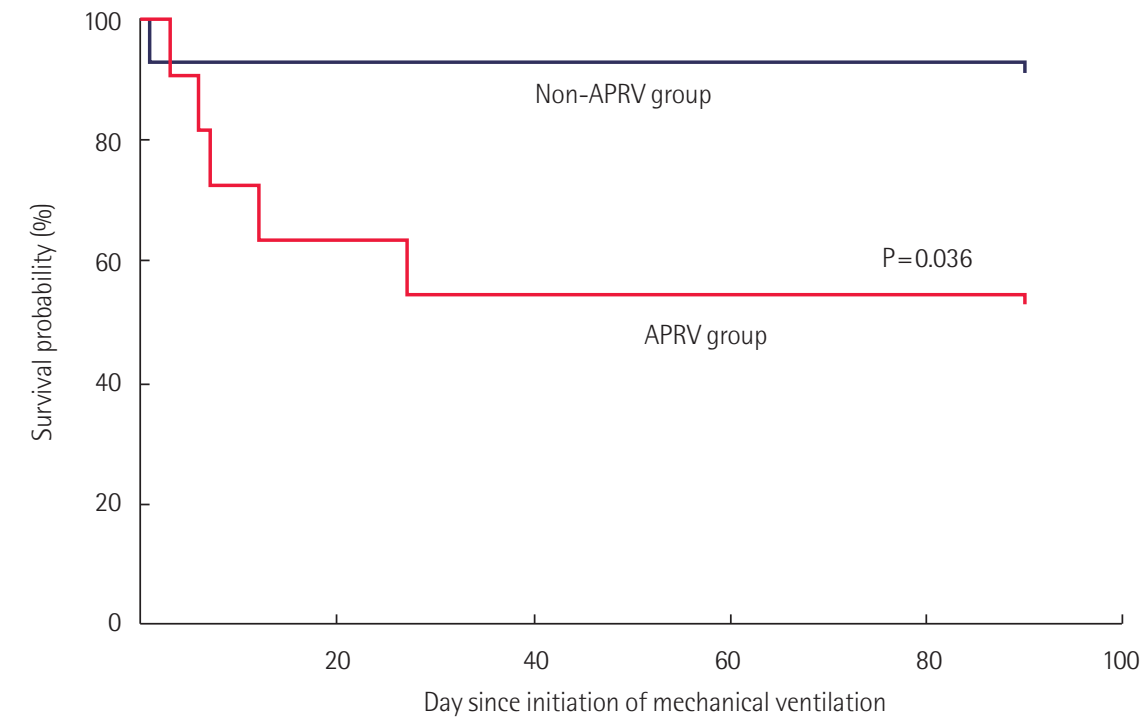
No. at risk
Non-APRV group
APRV group

$\begin{array}{rr}14 & 13 \\ 11 & 7\end{array}$

13

6

13

6

$\begin{array}{rl}13 & 0 \\ 6 & 0\end{array}$

Figure 2. Survival curves for patients treated and untreated with airway pressure release ventilation (APRV) during their intensive care unit stay $(n=25)$. The APRV group had a lower probability of survival to day 90 compared to the non-APRV group of mechanically ventilated patients (hazard ratio of survival, $0.17 ; 95 \%$ confidence interval, $0.03-0.89 ; \mathrm{P}=0.036$ ).

Table 4. Propensity score based on indices of severity of illness to predict use of APRV in all patients $(n=25)$

\begin{tabular}{|c|c|c|}
\hline Predictor & Odds ratio $(95 \% \mathrm{Cl})$ & P-value \\
\hline APACHE II score & $1.335(0.801-2.224)$ & 0.260 \\
\hline $\mathrm{PaO}_{2}$ to $\mathrm{FiO}_{2}$ ratio (on initiation of mechanical ventilation) & $0.906(0.776-1.059)$ & 0.215 \\
\hline Maximum CRP (per mg/L increment) & $1.082(0.986-1.189)$ & 0.097 \\
\hline Respiratory compliance (per $\mathrm{ml} / \mathrm{cm} \mathrm{H}_{2} \mathrm{O}$ increment) on initiation of mechanical ventilation & $1.116(0.882-1.412)$ & 0.362 \\
\hline No. of quadrants with infiltrates on the CXR on admission to ICU & $0.003(0.001-19.82)$ & 0.197 \\
\hline $\mathrm{FiO}_{2}$ & $0.870(0.700-1.082)$ & 0.210 \\
\hline
\end{tabular}

The Hosmer-Lemeshow chi-square and Nagelkerke $R^{2}$ of the model were $0.820(P=0.999)$ and 0.840 , respectively. Median and interquartile range of propensity scores for ICU survivors were 0.09 and $0.01-0.91$, respectively, and those for ICU non-survivors were 0.95 and 0.16-0.99.

APRV: airway pressure release ventilation; $\mathrm{Cl}$ : confidence interval; APACHE: Acute Physiology and Chronic Health Evaluation; $\mathrm{PaO}_{2}$ : arterial oxygen partial pressure; $\mathrm{FiO}_{2}$ : fractional inspired oxygen; $\mathrm{CRP}$ : C-reactive protein; $\mathrm{CXR}$, chest $\mathrm{X}$-ray.

\section{DISCUSSION}

In adult patients with COVID-19 requiring invasive mechanical ventilation in the ICU, APRV was associated with a significant reduction in 90-day survival compared to conventional ventilation. APRV was associated with significantly higher driving pressures, higher tidal volumes, and lower respiratory compliance than conventional ventilation. The association with lower survival remained significant in a multivariable propensity analysis. These findings are contrary to the hypothesis that APRV use would improve outcomes and, to our knowledge, are the first published findings of APRV use in COVID-19.
There is ongoing debate regarding the rate at which COVID-19 patients who are mechanically ventilated will progress to ARDS [14-18]. There is conflicting evidence regarding lung compliance in patients with COVID-19 pneumonitis, both with regard to magnitude of compliance and change in compliance over time [14-16,19]. The majority of studies on compliance in early COVID-19 pneumonitis have reported abnormally low lung compliance. Compliance measured in our cohort was similar to that seen in other groups [20]. This factor might serve as a confounding factor, as severe COVID-19 pneumonitis might not always behave like typical ARDS. In this context, the observed increased ventilator driving pressure of APRV might con- 
Table 5. Stepwise Cox proportional hazards regression to assess the effect of exposure to APRV on ICU mortality $(n=25)$ with and without adjusting for propensity to use APRV

\begin{tabular}{|c|c|c|c|c|}
\hline Covariate & $\begin{array}{l}\text { HR associated with APRV } \\
\text { in relation to survival to } \\
\text { ICU discharge }(95 \% \mathrm{Cl})\end{array}$ & P-value & $\begin{array}{l}\text { HR associated with APRV in } \\
\text { relation to survival to ICU } \\
\text { discharge }(95 \% \mathrm{CI}) \text { with } \\
\text { adjustment for propensity score }\end{array}$ & $\begin{array}{l}\text { P-value } \\
\text { (including } \\
\text { adjustment for } \\
\text { propensity score) }\end{array}$ \\
\hline APRV (yes vs. no) & $0.182(0.06-0.584)$ & 0.004 & $0.03(0.01-0.385)$ & 0.008 \\
\hline APRV+APACHE II score & $0.176(0.05-0.588)$ & 0.005 & $0.02(0.01-0.316)$ & 0.005 \\
\hline $\begin{array}{l}\mathrm{APRV}+\mathrm{APACHE} \| \mathrm{score}+\mathrm{PaO}_{2} / \mathrm{FiO}_{2} \text { ratio on initiation of } \\
\text { mechanical ventilation }\end{array}$ & $0.08(0.02-0.384)$ & 0.002 & $0.02(0.01-0.328)$ & 0.007 \\
\hline $\begin{array}{l}\mathrm{APRV}+\mathrm{APACHE} \| \mathrm{score}+\mathrm{PaO}_{2} / \mathrm{FiO}_{2} \text { ratio and respiratory } \\
\text { compliance on initiation of mechanical ventilation }\end{array}$ & $0.07(0.01-0.362)$ & 0.002 & $0.02(0.01-0.334)$ & 0.007 \\
\hline $\begin{array}{l}\mathrm{APRV}+\mathrm{APACHE} \| \mathrm{score}+\mathrm{PaO}_{2} / \mathrm{FiO}_{2} \text { ratio and respiratory } \\
\text { compliance on initiation of mechanical ventilation+ } \\
\text { corticosteroid therapy }\end{array}$ & $0.07(0.01-0.356)$ & 0.002 & $0.02(0.01-0.514)$ & 0.018 \\
\hline APRV+ & $0.06(0.01-0.359)$ & $0.002^{\mathrm{a}}$ & $0.02(0.01-0.554)$ & 0.020 \\
\hline APACHE II score+ & $0.88(0.73-1.06)$ & 0.164 & $0.89(0.73-1.08)$ & 0.238 \\
\hline $\mathrm{P} / \mathrm{F}$ ratio+ & $1.00(0.99-1.01)$ & 0.575 & $1.00(0.99-1.01)$ & 0.454 \\
\hline Respiratory compliance on initiation MV+ & $0.99(0.94-1.03)$ & 0.552 & $0.99(0.95-1.04)$ & 0.645 \\
\hline Corticosteroid therapy+ & $0.35(0.04-3.47)$ & 0.371 & $0.60(0.04-9.88)$ & 0.724 \\
\hline No quadrants on CXR+ & $1.39(0.47-4.16)$ & 0.554 & $1.30(0.42-3.96)$ & 0.650 \\
\hline Propensity score & Not included & - & $3.68(0.11-119)$ & 0.463 \\
\hline
\end{tabular}

APRV: airway pressure release ventilation; ICU: intensive care unit; HR: hazard ratio; Cl: confidence interval; APACHE: Acute Physiology and Chronic Health Evaluation; $\mathrm{PaO}_{2}$ : arterial oxygen partial pressure; $\mathrm{FiO}_{2}$ : fractional inspired oxygen; $\mathrm{CXR}$ : chest X-ray.

${ }^{a}$ None of the other covariates other than APRV was associated with a P-value $<0.05$; the HR for ICU survival $(0.06)$ and P-value $(0.002)$ associated with APRV remained unchanged after 1,000 bootstrap resamplings to adjust for bias from overfitting or outliers.

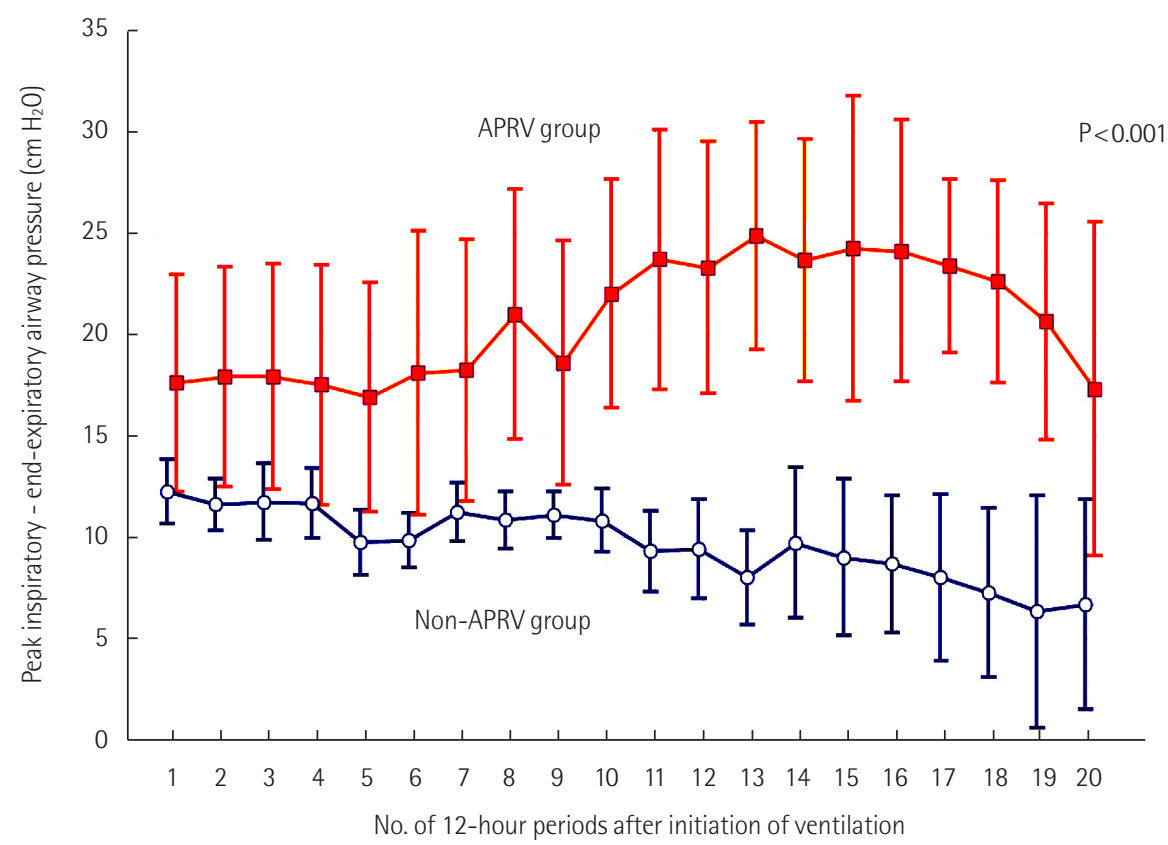

Figure 3. The difference in ventilating driving pressure (peak inspiratory minus end-expiratory airway pressure) from initiation of invasive mechanical ventilation until day 10 between those treated and untreated by airway pressure release ventilation (APRV). 


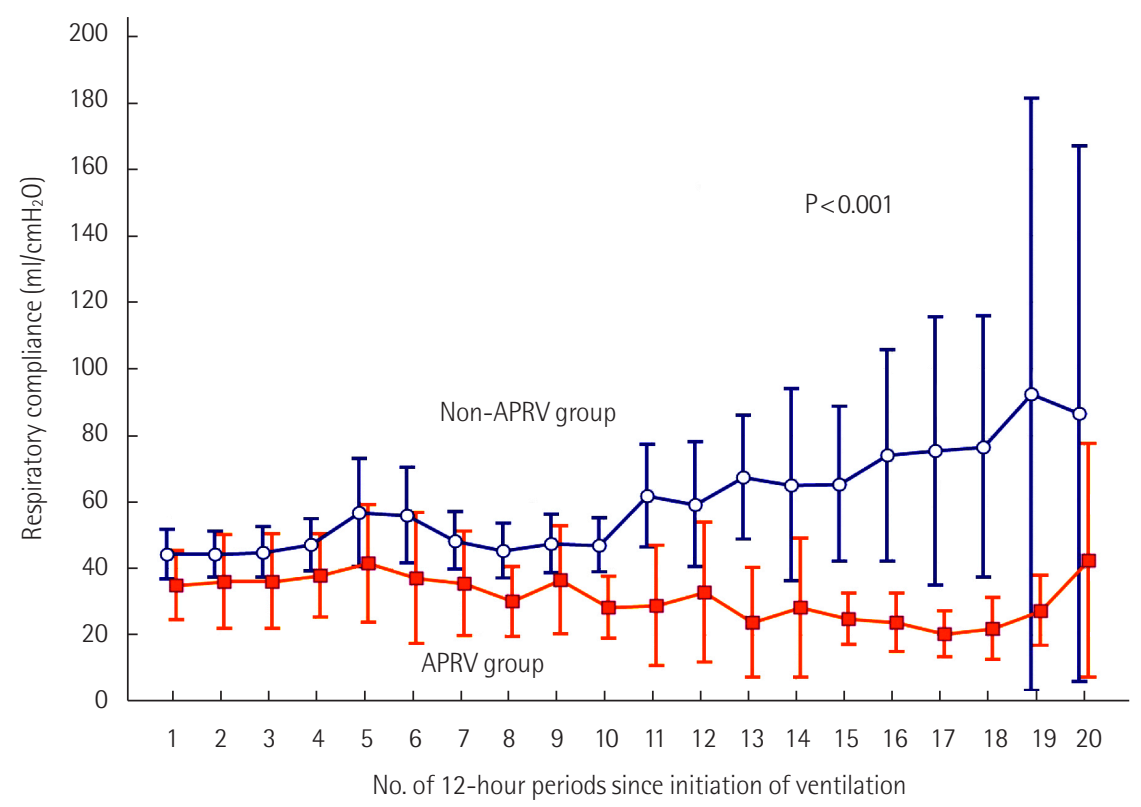

Figure 4. The difference in respiratory compliance from initiation of invasive mechanical ventilation until day 10 between those treated and untreated by airway pressure release ventilation (APRV).

tribute to ventilator-associated lung injury. This injury could be mitigated via strategies to reduce driving pressures.

There are several limitations to our study. This was a small, retrospective, cohort study. While we attempted to reduce confounding factors, there remains the possibility that the effect seen was due to the confounding effects of variables that we did not consider. While it does not appear that APRV was used solely as a rescue method of ventilation, it is possible that this was not detected due to the small sample size. Indeed, while not statistically significant, there was greater use of prone positioning and nitric oxide administration subsequent to initiation of APRV compared to that in the other group. The interquartile ranges for APACHE score, initial $\mathrm{PaO}_{2} / \mathrm{FiO}_{2}$ ratio, and initial compliance suggest that the APRV group was more unwell than the non-APRV group, despite the lack of statistical significance. A larger patient cohort would enable this to be explored in more detail.

COVID-19 pneumonitis is a novel disease process about which there are limited data available regarding use of invasive ventilation. Ventilation mode and settings were at the discretion of the treating intensivist. While APRV is provided as the usual care for severe hypoxic respiratory failure at three of the five sites involved, there was no standardization of ventilator settings used in APRV, or any form of ventilation, among sites. The higher driving pressures and wider interquartile ranges for the driving pressures seen in the APRV group suggest greater variability compared to the non-APRV group. It might be that the fibroproliferative phase of COVID-19 pneumonitis is worsened by this variability in driving pressures. As this was a retrospective study, ventilator settings were outside the control of this study. This variability in ventilator settings might be a major confounding factor in this study.

Based on the findings of this study, we would urge caution with the use of APRV in COVID-19 patients. Safety and efficacy need to be established by a large prospective observational trial or a randomised controlled trial. This can be assisted by a standardized approach to APRV in patients, with close attention paid to lung compliance, driving pressures, and tidal volume.

\section{CONFLICT OF INTEREST}

No potential conflict of interest relevant to this article was reported.

\section{ACKNOWLEDGMENTS}

This study was supported by a COVID-19 research grant from Department of Health, Western Australia.

We acknowledge Timothy Yap (Curtin Medical School), Anna-Maria Palermo (Fiona Stanley Hospital), Rashmi Rauniyar (Sir Charles Gairdner Hospital), Danielle Barton (Joondalup Hospital), and Sharon Waterson (Royal Perth Hospital) for their review of the clinical charts and David Hawkins (Joondalup Hospital) for his assistance. There was no financial com- 
pensation for these contributions.

\section{ORCID}

Kwok M. Ho https://orcid.org/0000-0002-6705-6004

Edward Litton https://orcid.org/0000-0002-5125-6829

Edward Fysh https://orcid.org/0000-0003-1126-3773

Matthew H. Anstey https://orcid.org/0000-0001-7927-524X

\section{AUTHOR CONTRIBUTIONS}

Conceptualization: JSZ, BW, EL, EF, MHA. Investigation: JSZ, KMH, EL, MHA. Data curation: JSZ. Formal analysis: KMH. Funding acquisition: MHA. Methodology: BW, MHA. Writing-original draft: JSZ, MHA. Writing-review \& editing: BW, $\mathrm{KMH}, \mathrm{EL}, \mathrm{EF}$.

\section{REFERENCES}

1. Coronaviridae Study Group of the International Committee on Taxonomy of Viruses. The species severe acute respiratory syndrome-related coronavirus: classifying 2019-nCoV and naming it SARS-CoV-2. Nat Microbiol 2020;5:536-44.

2. World Health Organization. Timeline of WHO's response to COVID-19 [Internet]. Geneva: World Health Organization; 2020 [cited 2020 Aug 29]. Available from: https://www.who. int/news-room/detail/29-06-2020-covidtimeline.

3. World Health Organization. Coronavirus disease (COVID-19) situation reports [Internet]. Geneva: World Health Organization; 2020 [cited 2020 Aug 29]. Available from: https://www. who.int/emergencies/diseases/novel-coronavirus-2019/situation-reports.

4. Bouadma L, Lescure FX, Lucet JC, Yazdanpanah Y, Timsit JF. Severe SARS-CoV-2 infections: practical considerations and management strategy for intensivists. Intensive Care Med 2020; 46:579-82.

5. Chen J, Qi T, Liu L, Ling Y, Qian Z, Li T, et al. Clinical progression of patients with COVID-19 in Shanghai, China. J Infect 2020;80:e1-e6.

6. Wu Z, McGoogan JM. Characteristics of and important lessons from the coronavirus disease 2019 (COVID-19) outbreak in china: summary of a report of 72314 cases from the Chinese center for disease control and prevention. JAMA 2020;323: 1239-42.

7. Docherty AB, Harrison EM, Green CA, Hardwick HE, Pius R, Norman L, et al. Features of 20133 UK patients in hospital with covid-19 using the ISARIC WHO clinical characterisa- tion protocol: prospective observational cohort study. BMJ 2020;369:m1985.

8. Potere N, Valeriani E, Candeloro M, Tana M, Porreca E, Abbate A, et al. Acute complications and mortality in hospitalized patients with coronavirus disease 2019: a systematic review and meta-analysis. Crit Care 2020;24:389.

9. Lim J, Litton E. Airway pressure release ventilation in adult patients with acute hypoxemic respiratory failure: a systematic review and meta-analysis. Crit Care Med 2019;47:1794-9.

10. Modrykamien A, Chatburn RL, Ashton RW. Airway pressure release ventilation: an alternative mode of mechanical ventilation in acute respiratory distress syndrome. Cleve Clin J Med 2011;78:101-10.

11. Zhou Y, Jin X, Lv Y, Wang P, Yang Y, Liang G, et al. Early application of airway pressure release ventilation may reduce the duration of mechanical ventilation in acute respiratory distress syndrome. Intensive Care Med 2017;43:1648-59.

12. Harris PA, Taylor R, Thielke R, Payne J, Gonzalez N, Conde JG. Research electronic data capture (REDCap): a metadata-driven methodology and workflow process for providing translational research informatics support. J Biomed Inform 2009; 42:377-81.

13. Harris PA, Taylor R, Minor BL, Elliott V, Fernandez M, O'Neal $\mathrm{L}$, et al. The REDCap consortium: Building an international community of software platform partners. J Biomed Inform 2019;95:103208.

14. Gattinoni L, Chiumello D, Rossi S. COVID-19 pneumonia: ARDS or not? Crit Care 2020;24:154.

15. Bos LD, Sinha P, Dickson RP. The perils of premature phenotyping in COVID-19: a call for caution. Eur Respir J 2020;56: 2001768.

16. Bos LD, Sinha P, Dickson RP. Response to COVID-19 phenotyping correspondence. Eur Respir J 2020;56:2002756.

17. Rajendram R. Building the house of CARDS by phenotyping on the fly. Eur Respir J 2020;56:2002429.

18. Ziehr DR, Alladina J, Petri CR, Maley JH, Moskowitz A, Medoff $\mathrm{BD}$, et al. Respiratory pathophysiology of mechanically ventilated patients with COVID-19: a cohort study. Am J Respir Crit Care Med 2020;201:1560-4.

19. Gattinoni L, Camporota L, Marini JJ. COVID-19 phenotypes: leading or misleading? Eur Respir J 2020;56:2002195.

20. Botta M, Tsonas AM, Pillay J, Boers LS, Algera AG, Bos LD, et al. Ventilation management and clinical outcomes in invasively ventilated patients with COVID-19 (PRoVENT-COVID): a national, multicentre, observational cohort study. Lancet Respir Med 2021;9:139-48. 